\title{
The validity and reliability of the Turkish brief version of the Zimbardo Time Perspective Inventory for adolescents
}

\author{
Ercan Kocayoruk $^{1}{ }^{(}$, Omer Faruk Simsek $^{2}$ \\ ${ }^{1}$ Canakkale Onsekiz Mart University, Department of Psychological Counseling and Guidance, Canakkale - Turkey \\ ${ }^{2}$ Rumeli University, Department of Psychology, Istanbul - Turkey
}

\begin{abstract}
Objective: The study aimed to examine the psychometric properties of the Zimbardo Time Perspective Inventory (ZTPI) developed by Zimbardo and Boyd with a sample of subjects in late adolescence (aged between 17 and 24 years).

Method: In order to determine how well the identified model of the shortened version of the ZTPI (five subscales with 15 items) fits the Turkish adaptation, exploratory factor analysis (EFA) and confirmatory factor analysis (CFA) were performed to understand the factor structure of ZTPI.

Results: The results of the initial CFA suggested that the original factor structure of the ZPTI shortened version with 15 items fit the data for adolescents' ratings of time perspective on the measures. The EFA revealed a five-factor structure for ZPTI with a shortened scale. CFA using competing models strategy also supported the five-factor structure. Convergent validity study yielded moderate correlations with other mental health indicators.

Conclusion: The findings of the current study provided a good starting point for further ZTPI developments in Turkish culture. It is proposed that a proper balance between past, present, and future are now considered preconditions for success, mental health, and personal happiness. Considering the findings of the current study, time perspective may be useful for evaluating the personality concerns and well-being of clients in counseling and therapy.
\end{abstract}

Keywords: Adolescents, psychometric properties of ZPTI, subjective well-being, time perspective

\section{INTRODUCTION}

Time perspective (TP) is described as a continual flow of personal and social experiences being assigned to temporal categories, or time frames, that help to give order, coherence, and meaning to past, present, and future events $(1,2)$. As social and emotional experiences are affected by a person's TP, it is possible to say that TP has a dynamic influence on many important judgments, decisions, and actions. In fact, the concept of TP is very broad and encompasses a wide range of conscious and unconscious emotional, behavioral, and cognitive processes. According to Zimbardo and Boyd (1), TP is a result of the unconscious processes of assigning personal and social experiences to particular temporal categories to give meaning and coherence to these experiences. On the other hand, TP can emerge intentionally both through intentional individual endeavors and clinical and counseling interventions.

How to cite this article: Kocayoruk E, Simsek OF. The validity and reliability of the Turkish brief version of the Zimbardo Time Perspective Inventory for adolescents. Dusunen Adam The Journal of Psychiatry and Neurological Sciences 2020;33:40-49.

Correspondence: Ercan Kocayoruk, Canakkale Onsekiz Mart University, Department of Psychological Counseling and Guidance, Canakkale - Turkey Phone: +90 2862174763 E-mail: kocayoruk@comu.edu.tr 
There has been an increase in the attention paid to research on the "balanced time orientation" most adaptive for individuals, allowing them to switch temporal frames flexibly between past, future, and present depending on situational demands, resource assessments, or personal and social appraisals (1). According to Holman and Silver (3), concerns about psychological and physical functioning are associated with being stuck in any of the specific temporal zones. Thus, balanced time has been defined as "the mental ability to switch effectively among TPs depending on task features, situational considerations, and personal resources, rather than be biased towards a specific TP that is not adaptive across situations" (1). The behavior of persons applying such a time orientation would, on average, be determined by a compromise, or balancing among the contents of meta-schematic representations of past experiences, present desires, and future consequences.

Many research findings reveal that $\mathrm{TP}$ and a proper balance between past, present, and future are associated with the preconditions for success, mental health, and personal happiness. For instance, van Beek et al. (4) demonstrated the relationship of TP with psychopathology such as depression and neuroticism. Similarly, TP has been found to be related with mental health $(5,6)$ and significantly associated with subjective well-being (7). Moreover, Barnett et al. (8) documented connections among TP, substance use, and abuse, while Sansone et al. (9) elucidated the relationships between future-orientation and smoking behavior. TP was used in many studies to explain or predict various forms of health behaviors, particularly dieting and exercising $(10,11)$ and relevance to quitting smoking (12). TP is also involved in diverse forms of psychosocial functioning (13), interpersonal relationships (14), and career decision-making (15).

The Zimbardo Time Perspective Inventory (ZTPI) $(1,16)$ is a well-known and comprehensively conceptualized tool for the assessment of TP. The conceptualization of TP by the ZTPI provides a measure of multiple time frames as individual temporal profiles and assesses the five dimensions of TP: Past-Positive (PP), Past-Negative (PN), Present-Fatalistic (PF), Present-Hedonistic (PH), and Future (FU). PP reflects a warm and embracing view of the past. PN reflects a negative, aversive view of the past. $\mathrm{PH}$ reflects a hedonistic and risk-taking perspective, and an attitude toward pleasure, connected with little regard for future consequences. PF shows an orientation of a fatalistic, helpless, and hopeless attitude toward life and the future.
FU reflects a point of view and thoughts regarding the future weighing the consequences of one's actions. This dimension of TP assumes that behavior is dominated by striving for future goals and rewards $(7,17,18)$. Furthermore, the ZTPI has been shown to be a useful assessment tool in many different areas, and numerous empirical studies have revealed that TP is a valuable predictor of various behaviors. For example, the findings of Zimbardo et al. (19) showed that risky driving is associated with a present-TP. Similarly, Keough et al. (20) found that the reported use of alcohol, drugs, and tobacco is also associated with a present-TP. In addition, the results of Apostolidis et al. (21) indicated that the reported use of cannabis is associated with a present-time orientation. It is evident that the concept of TP has an essential influence on many aspects of human behavior.

The validity and reliability of the ZTPI has been tested in many cultures, primarily in consulting and clinical applications, and the ZTPI has been translated and validated in multiple languages. The currently available versions include the Italian (22), French (23), Spanish (24), Czech (25), Russian (26), Greek (5), Swedish (27), Lithuanian (28) and Portuguese (29) versions. Some of these versions were tested in large representative samples, such as the Lithuanian $(n=1529)$ and Czech $(n=2030)$ versions. Finally, the ZTPI was scrutinized by cross-cultural comparisons: In a comprehensive study, Sircova et al. (30) examined crosscultural similarities and differences in TP involving samples from 24 countries. The findings of their study showed that similar views of the past, present, and future and important similarities across countries in TP exist. Furthermore, results also stated that five temporal orientations were invariant across many countries with diverse cultural traditions. The authors suggested a revised version of the ZTPI as the "gold standard" for further research on TP.

The validation studies showed that the translations of the ZTPI are useful tools in psychological practice and generally match the original factor structure. Recent studies frequently used the 15 -item version of the ZTPI because of an apparent need for a short form of the instrument. One short form of the ZTPI was developed by Zhang et al. (7), consisting of 15 items in five scales. Similarly, Kostal et al. (31) focused on short versions (ZTPI-short), comprising 3 items for each scale, 15 items in total (or 18, when Future-Negative is added). The authors aimed to test the psychometric properties of the abbreviated ZTPI and the optional balancing of the Future scale with its negative counterpart. Seven items of the inventories of Kostal et al. (31) overlapped. 
The authors claimed that several items from the version of Zhang et al. (7) appeared problematic when they were used in other studies or in other cultural contexts. In summary, although driven by a logic similar to Zhang's team, they opted for rigorous item selection from both the American and the international perspective and the use of inventories in translation. Kostal et al. (31) tried to avoid items that appeared problematic in various national contexts. There was also a difference in the layout of the inventories. Zhang et al. (7) presented the items in consecutive groups, according to each scale, whereas Kostal et al. (31) released the items from the factor groupings in a way similar to how they were presented in the original ZTPI (1) while additionally rotating the items during administration to prevent any artifacts caused by item location in the questionnaire.

In line with the tenets of TP studies, a number of studies have been undertaken to modify or adapt an instrument for measuring TP in Turkish culture that is consistent across cultures. For instance, Sahin et al. (32) examined the internal consistency, structural validity, and convergent validity of Turkish Adolescent Time Inventory-Time Attitude scores. The authors concluded that 5 subscales (Past Positive, Past Negative, Present Positive, Present Negative, and Future Positive) can be used with Turkish adolescents and that the Future Negative subscale needs to be revised and validated in this national context because of internal consistency estimates for Future Negative scores. Of the instruments existing to date, the theoretically driven ZTPI is the most widely used and psychometrically sound instrument. One study investigated the relationships between undergraduate students' TPs and boredom coping strategies (33). The authors used the ZTPI with 39 items after exploratory factor analysis (EFA) and confirmatory factor analysis (CFA). They concluded that the fivefactor solution with 39 items was the best option when compared to other numbers of factors, showing an acceptable fit to the data. A study suggested by Akırmak (34) states that 178 students was carried out to understand the extent to which individuals perceptions of self and of interpersonal relationships using the ZTPI with 56 items. Consistent with the study aim, deviation from balanced TP (i.e., DBTP scores) was calculated by subtracting each participant's measured TP scale scores. To date, a number of attempts have been made to modify or validate the original TP instrument; however, there is no specified form of TP instrument. In addition, researchers have been looking for shorter scales to make data collection more time-efficient in order to eliminate item redundancy and therefore reducing fatigue, frustration, and boredom associated with answering highly similar questions repeatedly (35). From this perspective, there are obvious advantages to a short measure, and its instrumental value is well accepted by many researchers as discussed above.

In conclusion, the ZTPI-Short is a valid and reliable instrument for assessing TP and is recommended for further use. The aim of this study was to translate the short version of the ZTPI into Turkish and examine its reliability and validity in a relatively large sample. For this objective, the short version of Kostal et al. (31), (ZTPI-Short), with 3 items for each scale (15 items in total) was utilized.

\section{METHOD}

\section{Study I: Factor Structure and Initial Reliability Participants}

University students in selected classes were provided with an explanatory research statement and their consent and voluntary participation were sought. An identical procedure was carried out in all studies. In study 1 , the sample consisted of 353 undergraduate students studying at Canakkale Onsekiz Mart University, which is a medium-sized higher education institute in Turkey. Eight participants with missing data were removed from the analyses. Thus, the final sample included 345 participants. The age in the sample consisting of 112 male (33\%) and 233 female students (67\%) ranged from 17 to 24 years (Mean $=20.74$, $\mathrm{SD}=4.09)$.

\section{Measure}

Short Version of the Zimbardo Time Perspective Inventory (ZTPI-Short): The ZTPI is the first comprehensive and theory-based operationalization of TP. The ZTPI, which was developed as an individualdifferences metric, assesses the fundamental dimensions of the human condition related to time (1). The scale is composed of 56 items. After exploratory and confirmatory factor analyses, five distinct TP factors emerged: PN, PP, PF, PH, and FU. The analyses showed acceptable validity and internal and test-retest reliability. In the current study, a short version of the ZTPI has been applied, using the version tested by Kostal et al. (31) both in its five-scale and six-scale forms (with and without the Future-Negative scale). The authors suggested that the five-scale ZTPI-Short form with 15 items had a slightly better model fit than the six-scale version, because the Future-Negative scale correlated strongly with the PN scale. Thus in our study, 15 items 
from the five-scale short form by Kostal et al. (31) (without the Future-Negative scale) were translated into Turkish. The authors reported that the internal consistency of the scales measured by Cronbach's alpha varied from 0.65 to 0.78 .

\section{Results}

\section{Exploratory Factor Analysis}

The factor structure of the ZPTI-Short version was explored using principal axis factor analysis with varimax rotation. In order to examine the factor structure of the ZPTI-Short version with 15 items, a common factor analysis with varimax rotation was conducted. The data and sample size were adequate for factor analysis according to Bartlett's test of sphericity, $\chi^{2}(105, \mathrm{n}=345)=1092.92, \mathrm{p}=0.001$, and the KaiserMeyer-Olkin measure of sampling adequacy (0.761). An unrotated five factor solution with an eigenvalue $\geq 1.0$ was obtained for the total sample, accounting for $62.29 \%$ of the variance. The first factor accounted for $20.17 \%$ of the variance (eigenvalue $=3.02$ ) and the second factor accounted for $12.85 \%$ of the variance (eigenvalue $=1.92$ ). Subsequent factors added similar rates to the explained variance $(11.03 \%$, eigenvalue $=1.65)$. The fourth factor accounted for 9.97\% (eigenvalue $=1.49$ ) and the last factor added $8.24 \%$ to the explained variance (eigenvalue $=1.23$ ). The results revealed that the factor solution grouped the 15 items into five factors. The basic psychometric properties of the ZTPI-Short are listed in Table 1.

In addition, the internal consistencies of the five factors were acceptable, with $\alpha=0.65$ for the first factor, $\alpha=0.61$ for the second factor, $\alpha=0.64$ for the third factor, $\alpha=0.66$ for the fourth factor, and $\alpha=0.79$ for the last factor. These Cronbach's alpha values are consistent with other ZPTI translations such as the Czech and Slovak study (from 0.65 to 0.78 ), the Lithuanian study (from 0.63 to 0.79 ), the Spanish (from 0.64 to 0.80 ), French (from 0.70 to 0.79 ), and the Swedish study (from 0.65 to 0.84$)$ (31).

\section{Study II: Confirmatory Factor Analysis Method}

\section{Strategy of Analysis}

CFA was implemented using a competing models strategy, and a series of first-order and higher-order CFA models were compared using both a chi-square difference test and information criterion indices such as Akaike Information Criterion (AIC) and Expected Cross Validation Index (ECVI). The following goodness-of-fit statistics were taken into consideration

\begin{tabular}{|c|c|c|c|c|c|c|c|}
\hline Items & Mean & SD & PN & PP & PF & PH & FU \\
\hline 1 & 3.95 & 0.93 & 0.73 & & & & \\
\hline 2 & 2.94 & 1.14 & 0.79 & & & & \\
\hline 3 & 3.44 & 1.02 & 0.73 & & & & \\
\hline 4 & 4.12 & 0.99 & & 0.73 & & 0.40 & \\
\hline 5 & 3.35 & 1.08 & & 0.71 & & & \\
\hline 6 & 4.14 & 0.85 & & 0.78 & & & \\
\hline 7 & 1.86 & 0.88 & & & 0.81 & & \\
\hline 8 & 1.61 & 0.85 & & & 0.81 & & \\
\hline 9 & 2.23 & 0.98 & & & 0.61 & & \\
\hline 10 & 4.06 & 0.72 & & & & 0.74 & \\
\hline 11 & 3.20 & 0.92 & 0.33 & & & 0.85 & \\
\hline 12 & 3.02 & 0.94 & & & & 0.71 & \\
\hline 13 & 4.04 & 0.96 & & & 0.31 & & 0.81 \\
\hline 14 & 3.45 & 1.04 & 0.40 & & & & 0.85 \\
\hline 15 & 3.59 & 0.98 & & 0.42 & & & 0.83 \\
\hline
\end{tabular}

Principal axis factor analysis with Direct-Oblimin method was used in the analyses; the ZTPI item ratings range from 1 to 5 . Likert scale anchors ranged from 1: not at all true to 5: extremely true. Factor loadings less than 0.30 are not represented; PN: Past-Negative, PP: Past-Positive, PF: Present-Fatalistic, PH: Present Hedonistic, FU: Future

to evaluate the fit of the data to the models. As index of the fit the Goodness of Fit Index (36) was employed with values greater than 0.90 indicating reasonable fit and greater than 0.95 indicating good fit, which is valid for the Comparative Fit Index (CFI). Root Mean Square Error of Approximation (37) was another statistical approach used, with values below 0.08 indicating reasonable fit of the model to the data and 0.05 indicating a good fit. The same criterion for reasonable fit is valid for the other goodness-of-fit statistics applied, the Standardized Root Mean Square.

\section{Participants}

Study 2 was conducted to confirm the factor structure of the ZTPI using confirmatory factor analyses. The sample consisted of 236 participants ranging in age from 18 to 24 years ( $63.6 \%$ females, $n=150 ; 36.4 \%$ male, $\mathrm{n}=86$ ).

\section{Results}

Confirmatory factor analyses were conducted using the competing models strategy (38). The original five-factor model was compared with two alternative models, as shown in Figure 1.

As can be seen from Figure 1, these models are the first-order one-factor model, first-order five-factor 


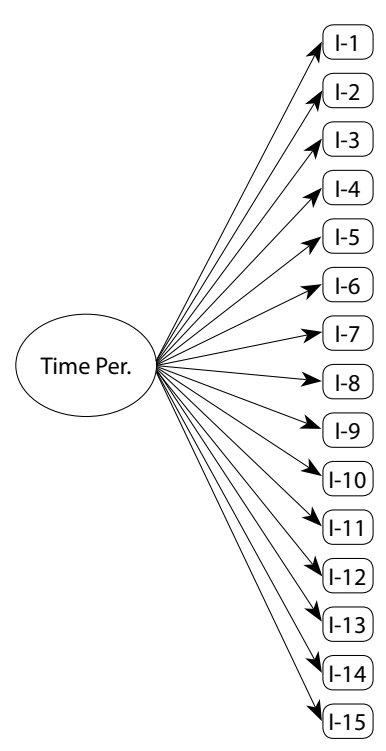

First-order one-factor model

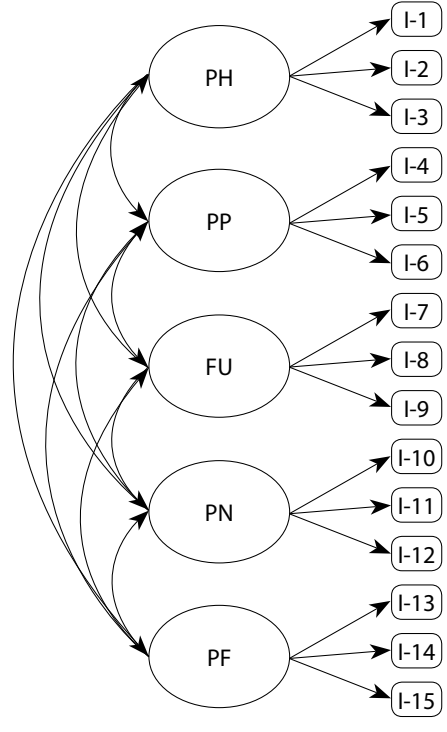

First-order five-factor model

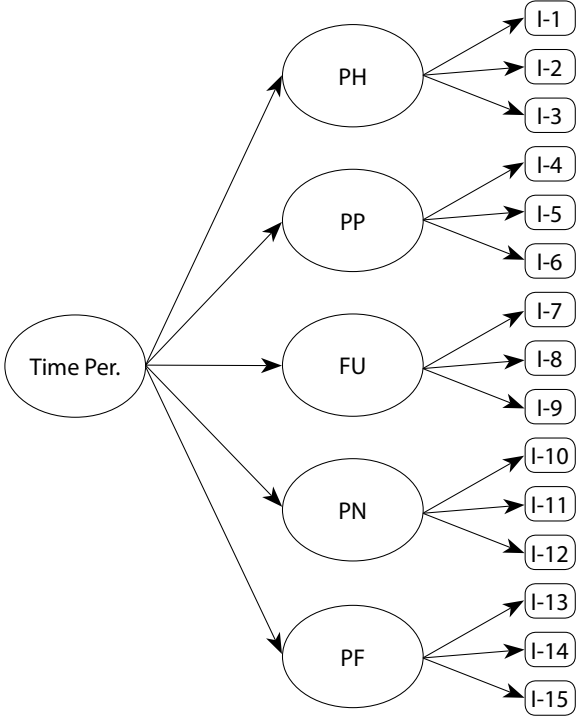

Higher-order one-factor model

Figure 1. Measurement models tested in the present study.

PH: Past hedonistic, PP: Past positive, FU: Future, PN: Past negative, PF: Present fatalistic.

model, and higher-order one-factor model. Goodness of Fit statistics calculated for these models are shown in Table 2.

According to the results obtained, the first-order five-factor model produced better results than the other higher-order models. This model was better than the higher-order one-factor model as indicated by the chisquare difference test $(19.22,5 ; \mathrm{p}<0.01)$. Smaller values of AIC and ECVI for the first-order five-factor model also confirmed that this model was better than the other models. This model was also better than the higherorder one-factor model as indicated by the significant chi-square difference test $(328.33,10 ; \mathrm{p}<0.01)$.
Intercorrelations among the factors of ZTPI-Short are shown in Table 3 , indicating weak or moderate relationships. It is clear from the table that the only strong relationship was between future and present fatalistic factors, indicating that having fatalistic opinions concerning the present has a strong relationship to have a negative outlook for future.

\section{Study III: Test-retest Reliability \\ Method}

\section{Participants}

The test-retest reliability measures were produced by correlating scores from time 1 and time 2. The sample

Table 2: Results of the Confirmatory Factor Analyses for the ZTPI-Short $(n=236)$

Indices

five-factor model

\begin{tabular}{lccc}
\hline$\chi^{2}$ & 134.54 & 153.76 & 462.87 \\
df & 80 & 85 & 90 \\
GFI & 0.93 & 0.92 & 0.79 \\
CFI & 0.93 & 0.91 & 0.54 \\
SRMR & 0.061 & 0.080 & 0.11 \\
RMSEA & 0.054 & 0.059 & 0.13 \\
& $(90 \% \mathrm{Cl}=0.038-0.070)$ & $(90 \% \mathrm{Cl}=0.044-0.073)$ & $(90 \% \mathrm{Cl}=0.12-0.14)$ \\
AIC & 215.54 & 223.76 & 522.87 \\
ECVI & 0.92 & 0.95 & 2.22 \\
\hline
\end{tabular}

GFI: Goodness-of-fit index, AGFI: Adjusted goodness-of-fit index, RMSEA: Root-mean-square error of approximation, CFI: Comparative fit index, $\mathrm{Cl}$ : Confidence interval 


\begin{tabular}{|c|c|c|c|c|}
\hline Factors & 1 & 2 & 3 & 4 \\
\hline 1 Past-negative & - & & & \\
\hline 2 Past-positive & $-0.34^{* *}$ & - & & \\
\hline 3 Present-fatalistic & $0.33^{* *}$ & -0.19 & - & \\
\hline 4 Present-hedonistic & -0.03 & 0.26 & -0.09 & - \\
\hline 5 Future & $0.48^{* *}$ & $-0.29 *$ & $0.76^{* *}$ & -0.11 \\
\hline
\end{tabular}

${ }^{*} p<0.05,{ }^{* *} p<0.01$

consisted of 149 university students (Mean=20.28; $\mathrm{SD}=1.79$ ), 100 females and 49 males randomly selected from the sample of Study 2. All students took part on a voluntary basis. The test-retest reliability was conducted over a 2-week interval. A meeting was held with the students for the retest procedure in which the scale was administrated collectively.

\section{Results}

The results of the Pearson's product-moment correlation coefficient revealed that test-retest reliability was moderate in the subscale. The results showed that the test-retest reliability was 0.55 for PN, 0.62 for PP, 0.57 for $\mathrm{PH}, 0.60$ for $\mathrm{PF}$, and 0.69 for FU.

\section{Study IV: Convergent Validity}

The aim of this study was to obtain evidence regarding the construct validity of the ZTPI by investigating its association with a number of scale scores related to personality traits and well-being. It was expected that the positive dimensions of the ZTPI subscale scores (FU, PH, and PP) would correlate positively with subjective well-being such as flourishing and the positive factors of ontological well-being in addition to positive dimensions of trait personality as extraversion, agreeableness, conscientiousness, and openness. At the same time, the negative dimensions of ZTPI subscale scores (PN and PF) were expected to correlate negatively with flourishing, the negative factors of ontological well-being, i.e., regret and nothingness, as well as negative dimensions of trait personality, namely, neuroticism.

\section{Method}

\section{Participants}

The sample for study IV consisted of 267 undergraduate students from Canakkale, Ankara, and Istanbul. The sample included an age range from 17 to 25 years (Mean=20.42, SD=2.09) and consisted of 72 males (27\%) and 195 females (73\%).

\section{Measures}

The Ontological Well-Being Scale (OWBS): The scale was developed to measure happiness and consisted of 24 items scored on a 5-point Likert-type scale (39). The OWBS is composed of four subscales: regret, activation, nothingness, and hope. The Regret subscale (7 items) assesses participants' feelings regarding the completed part of their life projects (the past). The Nothingness (6 items) and Activation (5 items) subscales measure feelings toward ongoing life projects (the present). Lastly, the Hope subscale (6 items) taps into the feelings about one's future life projects (the future). The original scale had good internal consistencies ranging from 0.78 to 0.90 , and the test-retest reliability of the OWBS, which was conducted over a 2-week interval, showed sufficient consistency of the scores over time (ranging from 0.72 to 0.92 ). In the current study, Cronbach's alpha values of OWBS varied from 0.61 to 0.73 .

The Big-Five Inventory (BFI): The 44-item BFI (40) was administered to assess five personality dimensions: Neuroticism, Extraversion, Openness, Agreeableness, and Conscientiousness. Ratings are indicated on a scale from 1 (disagree strongly) to 5 (agree strongly) for each item. The scale was adapted by Sumer et al. (41) who only reported Cronbach's alpha reliabilities ranging from 0.64 to 0.77 . The alpha coefficients were $0.75,0.82,0.67,0.51$, and 0.80 respectively in the data set used in this study.

Flourishing Scale (FS): FS is an 8-item scale that assesses features of human functioning such as positive relationships, feelings of competence, meaning and purpose in life, and engagement with daily activities (42). The 7-point Likert-type scale was used to evaluate the items, ranging from 1 (strongly disagree) to 7 (strongly agree). Higher scores on the scale indicate higher levels of well-being in important aspects of functioning and flourishing. The scale was adapted into Turkish by Akin and Fidan (43) who reported strong Cronbach's alpha reliability $(\alpha=0.89)$ for flourishing. The alpha coefficients were 0.86 in the data set used in the present study.

\section{RESULTS}

Intercorrelations of the OWBS scores with scores on the measures of well-being, trait personality, and motivation are presented in Table 4 . It is clear from Table 4 that five scales of ZPTI-Short have relatively acceptable correlations with the OWBS-subscale scores and the current dimensions of flourishing and personality. Two 


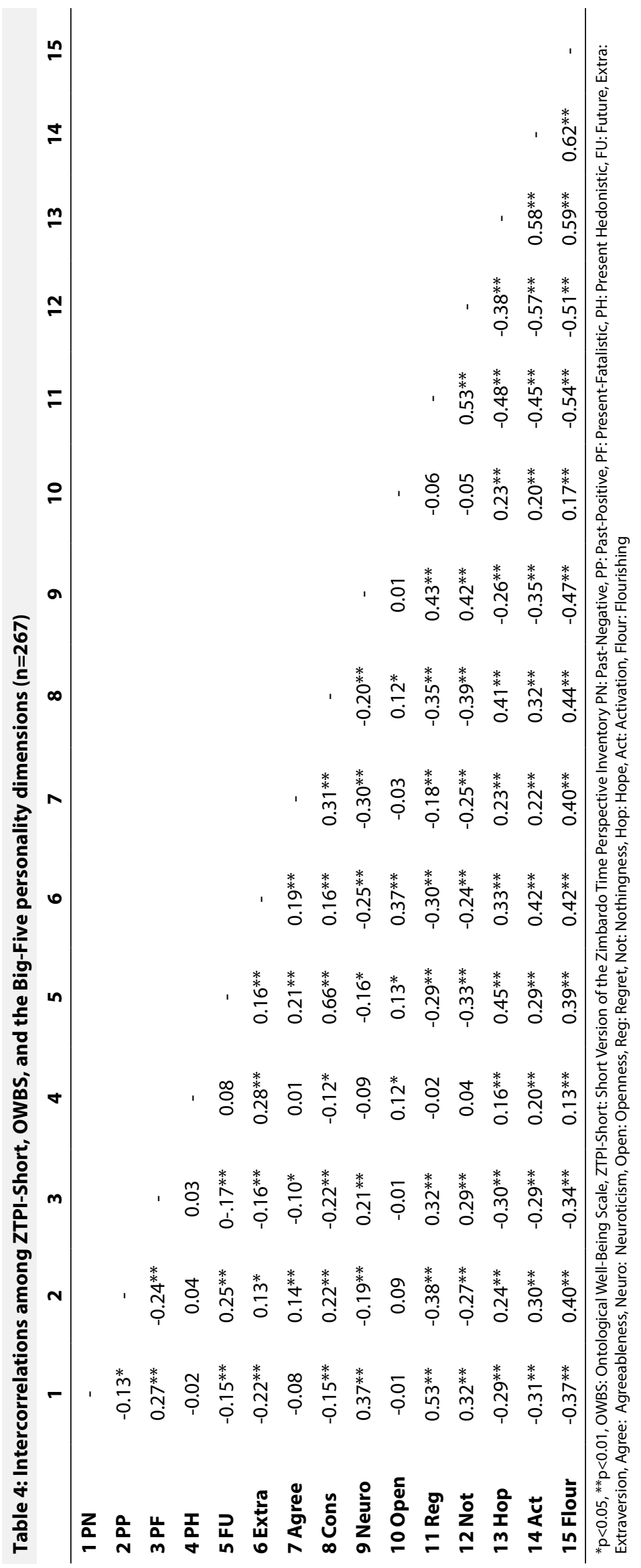

factors, 'regret' and 'nothingness' were positively correlated with $\mathrm{PN}$ and $\mathrm{PF}$, while 'activation' and 'hope' were correlated with PP and FU. As expected, 'regret' and 'nothingness' were negatively correlated with PP and F, while 'activation' and 'hope' were negatively correlated with PN and PF. Interestingly, PH was only positively correlated with 'activation,' 'hope' and 'flourishing.' On the other hand, FU was only correlated with all personality types and openness did not correlate with TP, except for $\mathrm{F}$.

\section{DISCUSSION}

The findings of confirmatory and EFA show that the five-factor structure of ZTPI (PN, PP, $\mathrm{PF}, \mathrm{PH}$ and $\mathrm{FU}$ ) as a 15 -item short form is validated with data from Turkish middle-age adolescents. Based on these results, it can be concluded that the ZTPI has an adequate validity, internal consistency, and reliability. With respect to convergent validity, the scale has considerably higher correlations with other measures of personality, life projects, and wellbeing. The results also show that an acceptable level of internal consistency is obtained for the five-factor structure and the findings are consistent with the theoretical framework with respect to convergent validity.

Increasing attention has been devoted to developing a short version of the ZTPI. Many studies reported that the original ZTPI with 56 items produced only an acceptable level of model fit (23); hence, a short version of the ZTPI promises a significant improvement for TP studies. Similarly, a short version of the five-factor structure of ZTPI with 15 items (without the Future-Negative scale) was recently published by Zhang et al. (7). The short version of ZTPI in Zhang's study suggested that 15 items in the five-factor structure (without the Future-Negative scale) had a good reliability, consistency and validity. Likewise, results in the present study showed that the short version of the ZTPI has very good psychometric properties and meets the good-fit criteria with regard to CFA in Turkish culture.

In terms of the ZTPI's dimensionality, the concluding structure is closely related to 
studies using the short form of ZTPI that have tended to derive structures consisting of five factors. For instance, Kostal et al. (31) tested both the five-scale and six-scale forms (with and without the FutureNegative scale). The results indicated that the fivescale ZTPI-Short had a better model fit in all indices compared to the six-scale ZTPI. Likewise, the number of significant factors obtained in the Swedish study conducted by Carelli et al. (27) concluded that the sixscale solution provided almost the same model fit as the original five-scale solution. Although goodness-offit statistics suggested a good or acceptable model fit, CFI values were quite low in the six-scale solution in their study. The authors claimed that correlations indicated strong relations among the Future-Negative and another negative scale, namely, Past-Negative.

In addition, the present study provides consistent relationships between TPs and personality traits and well-being, which are consistent with previous studies reporting consistent relationships between TPs and personality traits, life satisfaction, and positive and negative effects (44-46). The relationships among the scales provide consistent evidence to validate a short version of the ZTPI with 15 items. However, unlike the original study (1), the present study was conducted with a sample of undergraduate students. This raises the question of whether the short version of the ZTPI with 15 items could be applied to a nonstudent sample of adults. A related issue is that our data were collected specifically from undergraduate students in psychological counseling, where their specialized knowledge in psychology could play a role in influencing the outcome of the results. It would be advisable to replicate the short version of the ZTPI in a sample of adults.

Consequently, the short version of ZTPI could be suggested to assess the same multidimensional constructs in TP as those proposed by Zimbardo and Boyd (1). The findings of the current study provided a good starting point for further ZTPI and Balanced TP $(44,47)$ developments in Turkish culture. It is proposed that a proper balance between past, present, and future are now considered preconditions for success, mental health, and personal happiness $(1,17)$. Besides, the original ZTPI with 56 items may be too lengthy and time-consuming to be used in research, therapy, and counseling settings; by contrast, a more practical short version of the ZTPI could be easily administered in such settings to save time and to avoid placing an excessive burden on clients or research participants. Considering the findings of the current study, TP may be useful for evaluating personality concerns and well-being of clients in counseling and therapy.

The findings of the current study should be considered within several limitations. First, the study relied exclusively upon self-reported data from adolescents and students. Future analyses, therefore, should include informant reports from different age groups (i.e., adults, elderly) using a life-span point of view for TP as evidence for validity. Second, although correlation analyses were performed to evaluate the convergent and discriminant validity of the ZTPI-S with other psychological constructs, future analyses may also assess predictive validity by testing the potential causal effect of generalized expectancy on subsequent outcome variables that are theoretically linked to each of the temporal dimensions.

\begin{tabular}{|c|c|c|}
\hline \multicolumn{2}{|c|}{ Contribution Categories } & \multirow{2}{*}{$\begin{array}{l}\text { Author Initials } \\
\text { E.K., O.F.S. }\end{array}$} \\
\hline \multirow{3}{*}{ Category 1} & Concept/Design & \\
\hline & Data acquisition & E.K. \\
\hline & Data analysis/Interpretation & E.K., O.F.S. \\
\hline \multirow{2}{*}{ Category 2} & Drafting manuscript & E.K., O.F.S. \\
\hline & Critical revision of manuscript & N/A \\
\hline Category 3 & Final approval and accountability & E.K., O.F.S. \\
\hline \multirow{2}{*}{ Other } & Technical or material support & $\mathrm{N} / \mathrm{A}$ \\
\hline & Supervision & N/A \\
\hline
\end{tabular}

Ethics Committee Approval: This study approved by Canakkale Onsekiz Mart University Social Sciences and Educational Sciences Ethics Committee. (Date: 29.08.2014 No: 2014/19)

Informed Consent: Written informed consent of all patients was obtained.

Peer-review: Externally peer-reviewed.

Conflict of Interest: There is no any conflict of interest.

Financial Disclosure: TUBITAK, Grand: 115K417.

\section{REFERENCES}

1. Zimbardo PG, Boyd JN. Putting time in perspective: a valid, reliable, individual differences metric. J Pers Soc Psychol 1999; 77:1271-1288. [CrossRef]

2. Zimbardo P, Boyd J. The time paradox: The new psychology of time that can change your life. New York: Free Press, 2008.

3. Holman EA, Silver RC. Getting "stuck" in the past: temporal orientation and coping with trauma. J Pers Soc Psychol 1998; 74:1146-1163. [CrossRef]

4. van Beek W, Berghuis $\mathrm{H}$, Kerkhof A, Beekman A. Time perspective, personality and psychopathology: Zimbardo's time perspective inventory in psychiatry. Time and Society 2011; 20:364-374. [CrossRef]

5. Anagnostopoulos F, Griva F. Exploring time perspective in Greek young adults: validation of the Zimbardo Time Perspective Inventory and relationships with mental health indicators. Soc Indic Res 2012; 106:41-59. [CrossRef] 
6. Gilbert SP, Sifers SK. Bouncing back from a breakup: attachment, time perspective, mental health, and romantic loss. J College Stud Psychother 2011; 25: 295-310. [CrossRef]

7. Zhang JW, Howell RT, Bowerman T. Validating a brief measure of the Zimbardo Time Perspective Inventory. Time and Society 2013; 22;391-409. [CrossRef]

8. Barnett E, Spruijt-Metz D, Unger J, Rohrbac LA, Sun P, Sussman $\mathrm{S}$. Bidirectional associations between future time perspective and substance use among continuation high-school students. Subst Use Misuse 2013; 48:574-580. [CrossRef]

9. Sansone G, Fong GT, Hall PA, Guignard R, Beck F, Mons U, et al. Time perspective as a predictor of smoking status: findings from the International Tobacco Control (ITC) Surveys in Scotland, France, Germany, China, and Malaysia. BMC Public Health 2013; 13:346. [CrossRef]

10. Gellert P, Ziegelmann JP, Lippke S, Schwarzer, R. Future time perspective and health behaviors: Temporal framing of selfregulatory processes in physical exercise and dietary behaviors. Ann Behav Med 2012; 43: 208-218. [CrossRef]

11. Guthrie LC, Lessl K, Ochi O, Ward MM. Time perspective and smoking, obesity, and exercise in a community sample. Am J Health Behav 2013; 37:171-180. [CrossRef]

12. Hall PA, Fong GT, Yong HH, Sanson, G, Borland R, Siahpush M. Do time perspective and sensation-seeking predict quitting activity among smokers? Findings from the International Tobacco Control (ITC) Four Country Survey. Addict Behav 2012; 37: 1307-1313. [CrossRef]

13. Laghi F, Liga F, Baumgartner E, Baiocco R. Time perspective and psychosocial positive functioning among Italian adolescents who binge eat and drink. J Adolesc 2012; 35:1277-1284. [CrossRef]

14. Bernstein MJ, Benfield JA. Past perspective is related to present relationships: Past-positive and negative time perspectives differentially predict rejection sensitivity. Psychological Record 2013; 63:615-627. [CrossRef]

15. Taber BJ. Time perspective and career decision-making difficulties in adults. J Career Assess 2013; 21:200-209. [CrossRef]

16. Gonzales A, Zimbardo PG. Time in perspective: A Psychology Today survey report. Psychol Today 1985; 19:21-26.

17. Boniwell I, Zimbardo PG. Balancing time perspective in pursuit of optimal functioning: In Linley PA, Joseph S (editors). Positive Psychology in Practice. Hoboken NJ: John Wiley \& Sons, 2004, 165-178. [CrossRef]

18. Stolarski M, Matthews G, Postek S, Zimbardo PG, Bitner J. How we feel is a matter of time: Relationships between time perspectives and mood. J Happiness Stud 2014; 15:809-827.

19. Zimbardo PG, Keough KA, Boyd JN. Present time perspective as a predictor of risky driving. Pers Individ Dif 1997; 23:10071023. [CrossRef]

20. Keough KA, Zimbardo PG, Boyd JN. Who's smoking, drinking, and using drugs? Time perspective as a predictor of substance use. Basic Appl Soc Psych 1999; 21:149-164. [CrossRef]

21. Apostolidis T, Fieulaine N, Soule F. Future time perspective as predictor of cannabis use: exploring the role of substance perception among French adolescents. Addict Behav 2006; 31:2339-2343. [CrossRef]

22. D’Alessio M, Guarino A, De Pascalis V, Zimbardo PG. Testing Zimbardo's Stanford Time Perspective Inventory (STPI) - Short form: an Italian study. Time and Society 2003;12:334-347.

23. Apostolidis T, Fieulaine N. French validation of the Zimbardo Time Perspective Inventory (ZTPI). Eur Rev Appl Psychol 2004: 54:207-217. [CrossRef]

24. Diaz-Morales JF. Factorial structure and reliability of Zimbardo Time Perspective. Psicothema 2006; 18:565-571. (Spanish)

25. Lukavska K, Klicperova-Baker M, Lukavsky J, Zimbardo PG. ZTPI - Zimbardo Time Perspective Inventory. Cesk Psychol 2011; 55: 356-373. (Czech)

26. Mitina, O Sircova, A. Zimbardo Time Perspective Inventory (ZTPI): Results of Russian version psychometrics analysis. Moscow University Psychology Bulletin 2008; 7385. 67-89.

27. Carelli MG, Wiberg B, Wiberg M. Development and construct validation of the Swedish Zimbardo Time Perspective Inventory. Eur J Psychol Assess 2011; 27:220-227. [CrossRef]

28. Liniauskaite A, Kairys, A. The Lithuanian version of the Zimbardo time perspective inventory. Psichologija 2009; 40:6687. [CrossRef]

29. Milfont TL, Andrade PR, Belo RP, Pessoa VS. Testing Zimbardo Time Perspective Inventory in a Brazilian sample. Interam J Psychol 2008; 42:49-58.

30. Sircova, A, van de Vijver FJR, Osin E, Milfont TL, Fieulaine N, Kislali-Erginbilgic A, et al. A global look at time: a 24-country study of the equivalence of the Zimbardo Time Perspective Inventory. Sage Open 2014; 4:1-12. [CrossRef]

31. Kostal J, Klicperova-Baker M, Lukavska K, Lukavsky J. Short version of the Zimbardo Time Perspective Inventory (ZTPI - short) with and without the Future-Negative scale, verified on nationally representative samples. Time and Society 2016; 25:169-192. [CrossRef]

32. Sahin-Baltaci H, Tagay O, Worrell FC, Mello ZR. Psychometric properties of Turkish Adolescent Time Inventory-Time Attitude (ATI-TA) scores. International Perspectives in Psychology: Research, Practice, Consultation 2017; 6:47-59. [CrossRef]

33. Eren A. Coskun H. Time perspectives and boredom coping strategies of undergraduate students from Turkey. Educational Research for Policy and Practice 2015; 14:53-75. [CrossRef]

34. Akirmak U. How is time perspective related to perceptions of self and of interpersonal relationships? Span J Psychol 2014; 17.

35. Robins RW, Fraley RC, Roberts BW, Trzesniewski KH. A longitudinal study of personality change in young adulthood. J Pers 2001;69: 617-640. [CrossRef]

36. Joreskog KG, Sorbom D. Lisrel 8: Structural Equation Modeling with the SIMPLIS Command Language. Lincolnwood IL: Scientific Software International, 1993.

37. Steiger, J. H. Structural model evaluation and modification: An interval estimation approach. Multivariate Behav Res 1990; 25:173-180. [CrossRef] 
38. MacCallum RC, Wegener DT, Uchino BN, Fabrigar LR. The problem of equivalent models in applications of covariance structure analysis. Psychol Bull 1993; 114:185-199. [CrossRef]

39. Simsek OF, Kocayoruk E. Affective reactions to one's whole life: preliminary development and validation of the Ontological WellBeing Scale. J Happiness Stud 2013; 14:309-343. [CrossRef]

40. Benet-Martinez V, John OP. Los Cinco Grandes across cultures and ethnic groups: Multitrait-multimethod analyses of the Big Five in Spanish and English. J Pers Soc Psychol 1998; 75:729-750.

41. Sumer HC, Bilgic R, Sumer N, Erol T. Personality attributes as predictors of psychological well-being for NCOs. J Psychol 2005; 139:529-544. [CrossRef]

42. Diener E, Wirtz D, Tov W, Kim-Prieto C, Choi DW, Oishi S, et al. New well-being measures: short scales to assess flourishing and positive and negative feelings. Soc Indic Res 2010; 97:143156. [CrossRef]
43. Akin A, Fidan M. The validity and reliability of the Turkish version of the Flourishing Scale: In Third International Conference on New Trends in Education and their Implications (ICONTE) Istanbul, 2012, 26-28.

44. Boniwell I, Osin E, Alex LP, Ivanchenko GV. A question of balance: time perspective and well-being in British and Russian samples. J Posit Psychol 2010; 5:24-40. [CrossRef]

45. Dunkel CS, Weber JL. Using three levels of personality to predict time perspective. Curr Psychol 2010; 29:95-103. [CrossRef]

46. Zhang JW, Howell RT. Do time perspectives predict unique variance in life satisfaction beyond personality traits? Pers Individ Dif 2011; 50:1261-1266. [CrossRef]

47. Zhang JW, Howell RT, Stolarski M. Comparing three methods to measure a balanced time perspective: the relationship between a balanced time perspective and subjective well-being. J Happiness Stud 2013; 14:169-184. [CrossRef] 